\title{
Optimal Reconfiguration of Electrical Distribution Systems Using Mathematical Programming
}

\author{
Marlon C. O. Borges • John F. Franco • \\ Marcos J. Rider
}

Received: 1 April 2013 / Revised: 9 July 2013 / Accepted: 6 August 2013 / Published online: 28 August 2013

(C) Brazilian Society for Automatics-SBA 2013

\begin{abstract}
This paper presents a novel mixed integer nonlinear programming (MINLP) model for the reconfiguration problem of electrical distribution systems (EDS), aiming to reduce the active power losses of the system. Two linearization techniques are applied in the MINLP model to obtain an equivalent mixed integer linear programming (MILP) model. The use of an MILP model guarantees convergence to optimality using existing optimization software. The proposed MILP model represents, with a suitable degree of accuracy, the steady-state operation of the EDS. Considering the typical operating conditions of the EDS, additional constraints are included in the proposed MILP model to reduce the computational effort demanded by its solution. The results of four test systems $(33,70,136$, and 417 nodes) are presented in order to show the accuracy as well as the efficiency of the proposed solution technique.
\end{abstract}

Keywords Reconfiguration of electric power distribution systems - Active power losses reduction - Mixed integer linear programming

\section{List of symbols}

\section{Sets}

$\Omega_{\mathrm{b}} \quad$ Set of nodes

$\Omega_{1} \quad$ Set of branches

$\Omega_{\mathrm{c}} \quad$ Set of nodes that can be connected only by two branches

M. C. O. Borges · J. F. Franco - M. J. Rider $(\bowtie)$

Departamento de Engenharia Elétrica, Universidade Estadual Paulista (UNESP), Ilha Solteira, São Paulo, Brazil

e-mail: mjrider@dee.feis.unesp.br

\section{Constants}

$R_{i j} \quad$ Resistance of branch $i j(\Omega)$

$X_{i j} \quad$ Reactance of branch $i j(\Omega)$

$Z_{i j} \quad$ Impedance of branch $i j(\Omega)$

$\underline{V} \quad$ Minimum voltage magnitude $(\mathrm{kV})$

$\overline{\bar{V}} \quad$ Maximum voltage magnitude $(\mathrm{kV})$

$V^{\text {nom }} \quad$ Nominal voltage magnitude $(\mathrm{kV})$

$\bar{I}_{i j} \quad$ Maximum current magnitude of branch $i j(\mathrm{~A})$

$P_{i}^{\mathrm{D}} \quad$ Active power demand at node $i(\mathrm{~kW})$

$Q_{i}^{\mathrm{D}} \quad$ Reactive power demand at node $i$ (kVAr)

$\bar{\Delta}_{i j}^{\mathrm{S}} \quad$ Upper bound of each block of the power flow linearization of branch $i j$

$\bar{\Delta}^{\mathrm{V}} \quad$ Discretization step of $V_{i}^{\mathrm{sqr}}$

$m_{i j, w}^{\mathrm{S}} \quad$ Slope of the $w$ th block used in the linearization of the power flow of branch $i j$

$N \quad$ Number of nodes

$S \quad$ Number of discretizations used for the variable $V_{i}^{\text {sqr }}$

$W \quad$ Number of blocks of the piecewise linearization

\section{Variables}

$P_{i}^{\mathrm{G}} \quad$ Active power generated at node $i$

$Q_{i}^{\mathrm{G}} \quad$ Reactive power generated at node $i$

$Q_{i j} \quad$ Reactive power flow of branch $i j$

$P_{i j} \quad$ Active power flow in the branch $i j$

$V_{i}^{\text {sqr }} \quad$ Square of voltage magnitude at node $i$

$I_{i j}^{\text {sqr }} \quad$ Square of current flow magnitude of branch $i j$

$P_{i j}^{+}$

Active power flow in the branch $i j$ in the forward direction

$P_{i j}^{-} \quad$ Active power flow in the branch $i j$ in the backward direction

$Q_{i j}^{+} \quad$ Non-negative auxiliary variable to obtain $\left|Q_{i j}\right|$

$Q_{i j}^{-} \quad$ Non-negative auxiliary variable to obtain $\left|Q_{i j}\right|$ 


$\begin{array}{ll}y_{i j}^{+} & \begin{array}{l}\text { Binary variable that defines if the active power flow } \\ \text { is in the forward direction }\end{array} \\ y_{i j}^{-} & \begin{array}{l}\text { Binary variable that defines if the active power flow } \\ \text { is in the backward direction }\end{array} \\ x_{j, s} & \begin{array}{l}\text { Binary variable used in the discretization of } V_{j}^{\text {sqr }} \\ \Delta_{i j, w}^{\mathrm{P}}\end{array} \\ \Delta_{i j, w}^{\mathrm{Q}} & \begin{array}{l}\text { Value of the } w \text { th block associated with active power } \\ \text { flow of branch } i j\end{array} \\ a_{i j} & \begin{array}{l}\text { Value of the } w \text { th block associated with reactive } \\ \text { Auxiliary variable used in the modeling of the state }\end{array} \\ P_{i j, s}^{\mathrm{c}} & \begin{array}{l}\text { Poweranch } i j \\ V_{i}^{\mathrm{sqr}} I_{i j}^{\mathrm{sqr}}\end{array}\end{array}$

\section{Introduction}

The reconfiguration of electrical distribution systems (EDS) deals with the modification of the system topology by opening or closing interconnection switches located in strategic points of the system in order to isolate faults, improve voltage profile, or reduce the active power losses of the EDS. The optimal reconfiguration of an EDS is currently a relevant problem, especially when advanced distribution automation and smart grid technologies become more important and more accessible to distribution companies.

The reconfiguration of an EDS is a combinatorial problem, and it can be modeled as a mixed integer nonlinear programming (MINLP) problem (Lavorato et al. 2012) with the objective of minimizing the active power losses in the EDS, which is subject to various constraints, such as voltage magnitude limits on the nodes, current magnitude limits of branches, active and reactive power balances, and radial operation of the EDS. The dimension of the problem is directly related to the number of interconnection switches in the EDS. Therefore, if an EDS has $C$ switches, the number of possible topologies is $2^{C}$. Most of those topologies are not feasible, do not satisfy the radiality constraint, or have disconnected nodes (Schmidt et al. 2005).

One of the first technical solutions applied to the reconfiguration problem is the constructive heuristic algorithm proposed by Merlin and Back (1975). This work presented an integer programming model that allows the determination of high quality solutions. In 1996, Abur (1996) presented a formulation for the reconfiguration problem using a model for minimum active power flow cost; by ignoring the capacity limits of the branches, the author solved the reconfiguration problem using linear programming.

A more complete formulation for the reconfiguration problem was proposed in 2005 (Ramos et al. 2005), which modeled both topological and electrical constraints, making the model more realistic than previous proposals. The authors solved the model using two different optimization techniques: a genetic algorithm and a solver for mixed integer linear programming (MILP), in which the objective function and the electrical constraints were previously transformed into linear functions. In 2010, a mixed integer quadratic programming model for the reconfiguration problem was developed (Romero-Ramos et al. 2010), with the advantage of avoiding nonlinear constraints and additional binary variables.

Jabr et al. (2012) presented two models for the EDS reconfiguration problem. One of them used mixed integer conic programming to represent the reconfiguration of the network in a convex model. The second model was an MILP formulation, based on a polyhedral representation of the conic constraints used in the first model.

The combinatorial nature of the problem led the researchers to develop heuristic methods, such as the one developed by Baran and Wu (1989), which uses branch exchange and applies two specific methods for calculating the load flow in radial networks. In 1992, another heuristic was developed by Goswami and Basu (1992), which follows the technique of closing an interconnection switch to form a single loop in the system; then, the power flow is calculated to determine the branch of the loop with the lowest flow. Lastly, the interconnection switch associated with that branch is opened. This process is performed until the algorithm finds a configuration with minimum power losses.

In 2008, a new algorithm for the reconfiguration problem was proposed by Martin and Gil (2008). This algorithm begins with a radial topology by opening all switches that form loops. In each step, an interconnection switch is closed, and the heuristic procedure establishes a reduced number of candidate interconnection switches to be opened in order to eliminate the generated loop. Finally, the choice of the most effective switch for minimizing the power losses is obtained from a power flow calculation. In the same year, another algorithm was presented (Raju and Bijwe 2008), which begins with all interconnection switches closed, and in each step, sensitivities are used to determine the interconnection switch that is open, a step that is repeated until a radial configuration is obtained. In a second phase, the algorithm of branch exchange is applied to improve the quality of the solution. Abul'Wafa (2011) developed another heuristic algorithm, which used the branch exchange technique and a load flow algorithm based on graph theory. The load flow used makes it possible to reduce the interconnection switching combinations investigated.

Metaheuristics are also used to address the problem of reconfiguration, including genetic algorithms (Zhu 2002), simulated annealing (Chiang and Jean-Jumeau 1990), ant colony search (Chang 2008), and tabu search algorithms (Guimarães et al. 2004), among others. Other researchers have solved the problem of reconfiguration using artificial 
intelligence techniques such as fuzzy logic (Kangan and Barioni 1999) and artificial neural networks (Hayashi et al. 1996; Salazar et al. 2006).

If the reconfiguration problem is represented using an MINLP model, the solution technique does not guarantee that the configuration found is actually the optimal solution of the reconfiguration problem due to the non-convexity of the model; the same situation is repeated when heuristics, metaheuristics, and artificial intelligence are employed. In this way, an interesting alternative is to represent the EDS reconfiguration problem as an MILP, considering the efficiency of existent solvers for these problems and the fact that in this case, it can be ensured that the solution is actually the optimal one. With the evolution of optimization software and the increasing use of powerful solvers, some researchers (Lavorato et al. 2012; Jabr et al. 2012; Romero-Ramos et al. 2010) have developed mathematical models for the EDS reconfiguration problem.

In this work, an MINLP model for the EDS reconfiguration problem is proposed. Linearizations were made to obtain an equivalent MILP model. The use of an MILP model guarantees convergence to optimality using existing optimization software. The proposed model was tested in systems of 33, 70,136 , and 417 nodes. In order to validate the approximations performed, the steady-state operation point for the solutions was compared to that obtained using the load flow sweep method.

The main contributions of this paper are as follows:

(1) A novel MINLP model for the reconfiguration problem of EDS.

(2) An equivalent model of MILP for the EDS reconfiguration problem that presents an efficient computational behavior with conventional MILP solvers.

(3) Additional constraints are included in the proposed MILP model to reduce the computational effort demanded by its solution.

\section{A Novel MINLP Model for EDS Reconfiguration Problem}

Aiming to represent the steady-state operation of an EDS, the following assumptions are made, commonly used in load flow formulations (Shirmohammadi et al. 1988), and are shown in Fig. 1.

- The load on the EDS is represented as constant active and reactive power.

- The EDS is balanced and represented by its single phase equivalent.

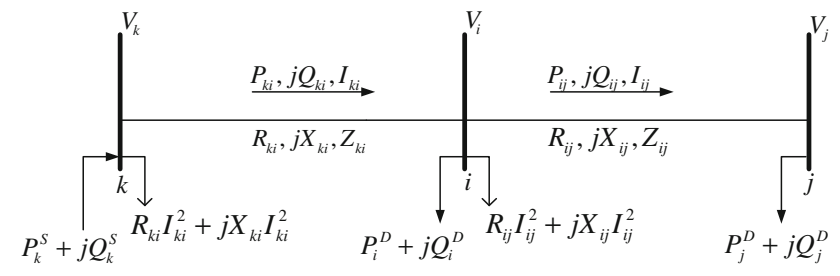

Fig. 1 Radial distribution system

With the assumptions described above, the reconfiguration problem of electrical distribution systems can be represented using an MINLP model, as shown in (1)-(15).

$\min v=\sum_{i j \in \Omega_{l}} R_{i j} I_{i j}^{\mathrm{sqr}}$

subject to

$\sum_{k i \in \Omega_{1}} P_{k i}-\sum_{i j \in \Omega_{l}}\left(P_{i j}+R_{i j} I_{i j}^{\mathrm{sqr}}\right)+P_{i}^{\mathrm{S}}=P_{i}^{\mathrm{D}} \quad \forall i \in \Omega_{\mathrm{b}}$

$\sum_{k i \in \Omega_{1}} Q_{k i}-\sum_{i j \in \Omega_{1}}\left(Q_{i j}+X_{i j} I_{i j}^{\mathrm{sqr}}\right)+Q_{i}^{\mathrm{S}}=Q_{i}^{\mathrm{D}} \quad \forall i \in \Omega_{\mathrm{b}}$

$$
\begin{array}{lcl}
V_{i}^{\mathrm{sqr}}-2\left(R_{i j} P_{i j}+X_{i j} Q_{i j}\right)- & \forall i j \in \Omega_{1} & \\
Z_{i j}^{2} I_{i j}^{\mathrm{sqr}}-V_{j}^{\mathrm{sqr}}-a_{i j}=0 & \forall i j \in \Omega_{1} & \\
V_{j}^{\mathrm{sqr}} I_{i j}^{\mathrm{sqr}}=P_{i j}^{2}+Q_{i j}^{2} & \forall i \in \Omega_{\mathrm{b}} & \\
\underline{V}^{2} \leq V_{i}^{\mathrm{sqr}} \leq \bar{V}^{2} & \forall i j \in \Omega_{1} & \\
I_{i j}^{\mathrm{sqr}} \leq \bar{I}_{i j}^{2}\left(y_{i j}^{+}+y_{i j}^{-}\right) & \forall i j \in \Omega_{1} & \\
P_{i j}^{+}-P_{i j}^{-}=P_{i j} & \forall i j \in \Omega_{1} & \\
0 \leq P_{i j}^{+} \leq \overline{V I}_{i j} y_{i j}^{+} & \forall i j \in \Omega_{1} & \\
0 \leq P_{i j}^{-} \leq \overline{V I}_{i j} y_{i j}^{-} & \forall i j \in \Omega_{1} \\
-\overline{V I}_{i j}\left(y_{i j}^{+}+y_{i j}^{-}\right) \leq Q_{i j} \leq \overline{V I}_{i j}\left(y_{i j}^{+}+y_{i j}^{-}\right) & \forall i j \in \Omega_{1} \\
\left|a_{i j}\right| \leq\left(\bar{V}^{2}-\underline{V}^{2}\right)\left(1-\left(y_{i j}^{+}+y_{i j}^{-}\right)\right) & \forall i j \in \Omega_{1} \\
\sum_{i j \in \Omega_{l}}\left(y_{i j}^{+}+y_{i j}^{-}\right)=N-1 & \forall i j \in \Omega_{1} \\
y_{i j}^{+}+y_{i j}^{-} \leq 1 & \forall i j \in \Omega_{1} .
\end{array}
$$

In the proposed model, it is assumed that all branches have an interconnection switch that can be opened or closed in order to obtain the best topology for the EDS operation. This is realized in order to demonstrate the efficiency and robustness of the proposed model using test systems as shown in Sect. 4.

The objective function (1) represents the active power losses of the EDS. Constraints (2) and (3) correspond to the balance of active and reactive power, respectively. The voltage magnitude drop in the branches is represented by (4), and the current flow magnitude calculation is expressed by (5). 
Equation (4) is similar to the related one shown in Cespedes (1990), but an additional variable $\left(a_{i j}\right)$ was added. This additional variable depends on the state of the interconnection switches. This equation allows to obtain the voltage magnitude of the final node $\left(V_{j}\right)$ in terms of the voltage magnitude of the initial node $\left(V_{i}\right)$, the real power flow $\left(P_{i j}\right)$, the reactive power flow $\left(Q_{i j}\right)$, the current magnitude $\left(I_{i j}\right)$, and the electrical parameters of branch $i j$. The voltage magnitude limits and the current flow capacity are represented by (6) and (7), respectively. The active power flow of the EDS is represented using two variables in (8), to identify the active power flow in the forward direction $(i j)$ and the backward direction (ji) with the variables $P_{i j}^{+}$and $P_{i j}^{-}$, respectively. These variables are bounded by (9) and (10) and can be used to include additional constraints in the model as shown in Sect. 3.2. The reactive power flow is limited by (11). The auxiliary variable $a_{i j}$ is zero if the branch is active $i j$ according to (12); otherwise, $a_{i j}$ is free within the limits defined by (12) in order to maintain and satisfy (4).

Constraint (13) is necessary (although not sufficient) to ensure radial configurations. Constraint (13), combined with (2) and (3), which represents the Kirchhoff's First Law (KFL), is used to obtain a radial topology for the reconfiguration problem, like shown in (Lavorato et al. 2012). Constraint (14) defines that only one binary variable related to the power flow direction is active.

Equation (15) represents the binary nature of the operation state of the interconnection switch of branch $i j$. In the model's Eqs. (1)-(15), two binary variables $\left(y_{i j}^{+}\right.$and $\left.y_{i j}^{-}\right)$ are used to represent the state of a branch due to its computational efficiency compared to the solution of a model that employs only one binary variable; this comparison was achieved experimentally. Additionally, these variables can be used to include additional constraints in the model as shown in Sect. 3.2. The binary variables $y_{i j}^{+}$and $y_{i j}^{-}$are associated with the direction of active power flow. $y_{i j}^{+}$is related to the $i j$ direction, while $y_{i j}^{-}$is related to the $j i$ direction. If both variables are zero, we obtain that the interconnection switch of the branch is open. If one of the variables has a value of 1 , it means that the branch is active, which means the interconnection switch is closed.

\section{Novel MILP Model for EDS Reconfiguration Problem}

\subsection{Linearization}

The model (1)-(15) is nonlinear because (5) presents the product of two variables $\left(V_{j}^{\mathrm{sqr}} I_{i j}^{\mathrm{sqr}}\right)$ and square terms $\left(P_{i j}^{2}\right.$ and $Q_{i j}^{2}$ ). Two linearization techniques are applied in (5) to obtain an equivalent MILP model; more information about these linearizations is shown in Franco et al. (2013a,b). Thus, to solve

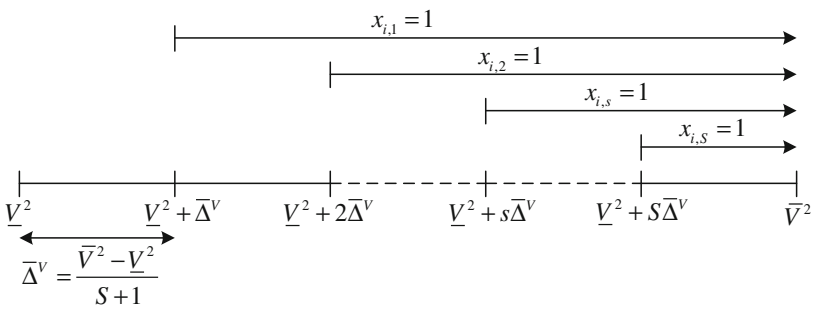

Fig. 2 Discretization of square of voltage magnitude

the EDS reconfiguration problem with an MILP model, it is necessary to replace the nonlinear Eq. (5) by linear equivalent constraints (16)-(17) and (19)-(20).

The left member of (5) is linearized through the discretization of $V_{j}^{\mathrm{sqr}}$ using the binary variables $x_{j, s} \forall s=0 \ldots S$, in which $x_{j, s}=1$ if $V_{j}^{\mathrm{sqr}}$ is greater than $V_{j}^{\mathrm{sqr}}$, as shown in Fig. 2 and explained in Franco et al. (2013b). The state of the variables $x_{j, s}$ is modeled in (16), with $\bar{\Delta}^{\mathrm{V}}=\frac{\left(\bar{V}^{2}-V^{2}\right)}{(S+1)}$.

$$
\begin{aligned}
& \underline{V}^{2}+\bar{\Delta}^{\mathrm{V}} \sum_{s=1}^{S} x_{j, s} \leq V_{j}^{\mathrm{sqr}} \\
& \leq \underline{V}^{2}+\bar{\Delta}^{V}+\bar{\Delta}^{V} \sum_{s=1}^{S} x_{j, s} \quad \forall i \in \Omega_{\mathrm{b}} \\
& x_{j, s} \leq x_{j, s-1} \quad \forall j \in \Omega_{\mathrm{b}}, s=1, \ldots, S \\
& x_{j, s} \in\{0,1\} \quad \forall j \in \Omega_{\mathrm{b}}, s=0, \ldots, S .
\end{aligned}
$$

The product $V_{j}^{\mathrm{sqr}} I_{i j}^{\mathrm{sqr}}$ is approximated using the middle point of the first interval of the discretization of the square voltage magnitude multiplied by the square current flow magnitude, plus the successive power corrections $\left(P_{i j, s}^{\mathrm{c}}\right)$, which depend on $\bar{\Delta}^{V}, \bar{V}, I_{i j}^{\mathrm{sqr}}$, and $x_{j, s}$ according to (17)

$$
\begin{aligned}
& -\bar{\Delta}^{V} \bar{V}^{2} \bar{I}_{i j}^{2}\left(1-x_{j, s}\right) \leq P_{i j, s}^{\mathrm{c}}-\quad \forall i j \in \Omega_{1}, s=0, \ldots, S \\
& \bar{\Delta}^{V} I_{i j}^{\mathrm{sqr}} \leq \bar{\Delta}^{V} \bar{V}^{2} \bar{I}_{i j}^{2}\left(1-x_{j, s}\right) \\
& 0 \leq P_{i j, s}^{c} \leq \bar{\Delta}^{V} \bar{V}^{2} \bar{I}_{i j}^{2} x_{j, s} \quad \forall i j \in \Omega_{1}, s=0, \ldots, S .
\end{aligned}
$$

Using (16) and (17), we can write (18), which approximates the left-hand side of (5).

$$
V_{j}^{\mathrm{sqr}} I_{i j}^{\mathrm{sqr}}=\left(\underline{V}^{2}+\frac{1}{2} \bar{\Delta}^{V}\right) I_{i j}^{\mathrm{sqr}}+\sum_{s=1}^{S} P_{i j, s}^{c} \quad \forall i j \in \Omega_{l} .
$$

The right member (5) is linearized as described in Franco et al. (2013a) and defined in Eqs. (19)-(20).

$$
\begin{aligned}
& \left(\underline{V}^{2}+\frac{\bar{\Delta}^{V}}{2}\right) I_{i j}^{\mathrm{sqr}}+\sum_{S=1}^{S} P_{i j, s}^{c}= \\
& \sum_{w=1}^{W} m_{i j, w}^{S} \Delta_{i j, w}^{P}+\sum_{w=1}^{W} m_{i j, w}^{S} \Delta_{i j, w}^{Q} \quad \forall i j \in \Omega_{1}
\end{aligned}
$$




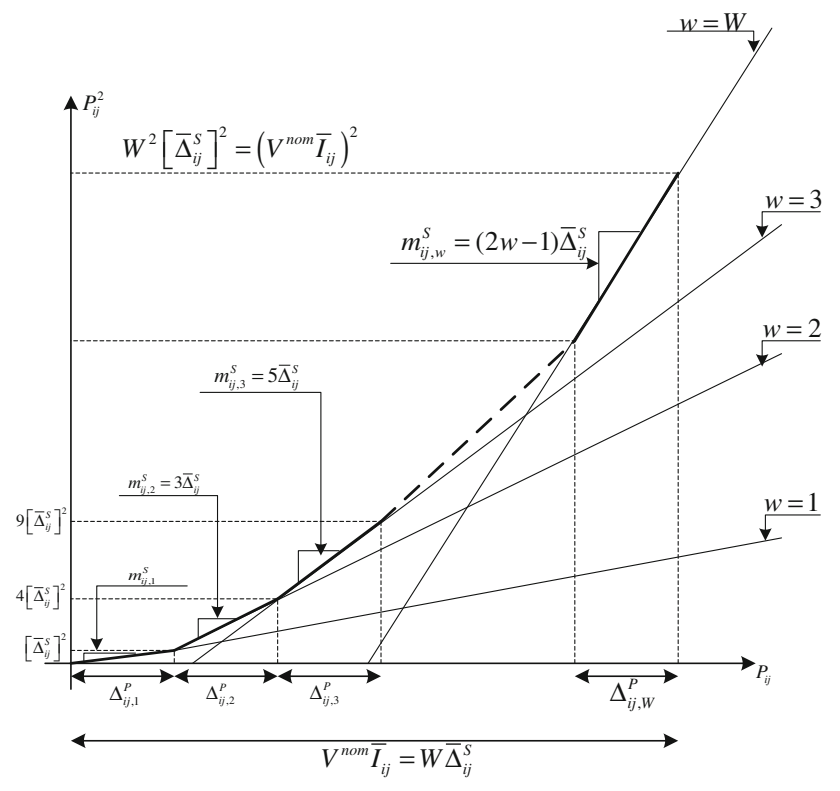

Fig. 3 Illustration of piecewise linear modeling of $P_{i j}^{2}$

$$
\begin{array}{ll}
Q_{i j}^{+}-Q_{i j}^{-}=Q_{i j} & \forall i j \in \Omega_{1} \\
P_{i j}^{+}+P_{i j}^{-}=\sum_{w=1}^{W} \Delta_{i j, w}^{P} & \forall i j \in \Omega_{1} \\
Q_{i j}^{+}+Q_{i j}^{-}=\sum_{w=1}^{W} \Delta_{i j, w}^{Q} & \forall i j \in \Omega_{1} \\
0 \leq \Delta_{i j, w}^{P} \leq \bar{\Delta}_{i j}^{S} & \forall i j \in \Omega_{1}, w=1, \ldots, W \\
0 \leq \Delta_{i j, w}^{Q} \leq \bar{\Delta}_{i j}^{S} & \forall i j \in \Omega_{1}, w=1, \ldots, W \\
0 \leq Q_{i j}^{+} & \forall i j \in \Omega_{1} \\
0 \leq Q_{i j}^{-} & \forall i j \in \Omega_{1},
\end{array}
$$

in which the constant parameters $m_{i j, w}^{S}$ and $\bar{\Delta}_{i j}^{S}$ are defined by

$$
\begin{array}{rlrl}
m_{i j, w}^{\mathrm{S}} & =(2 w-1) \bar{\Delta}_{i j}^{\mathrm{S}} & & \forall i j \in \Omega_{1}, w=1, \ldots, W \\
\bar{\Delta}_{i j}^{S} & =\frac{\overline{V I}_{i j}}{W} & \forall i j \in \Omega_{l} .
\end{array}
$$

The expressions $\sum_{w=1}^{W} m_{i j, w}^{\mathrm{S}} \Delta_{i j, w}^{\mathrm{P}}$ and $\sum_{w=1}^{W} m_{i j, w}^{\mathrm{S}} \Delta_{i j, w}^{\mathrm{Q}}$ are the linear approximations of $P_{i j}^{2}$ and $Q_{i j}^{2}$, respectively. The linearization of $P_{i j}^{2}$ is shown in Fig. 3. $Q_{i j}^{+}$and $Q_{i j}^{-}$are non-negative auxiliary variables to obtain $\left|Q_{i j}\right|$ as shown in (20a). Constraints (20b) and (20c) state that $\left|P_{i j}\right|$ and $\left|Q_{i j}\right|$ are equal to the sum of the values in each block of the discretization. Constraints (20d) and (20e) set the upper and lower limits of the contribution of each block of $\left|P_{i j}\right|$ and $\left|Q_{i j}\right|$.

Thus, the model defined by (1)-(4), (6)-(17), and (19)(20) is an MILP model that represents the EDS reconfigura- tion problem. Some constraints may be added to the proposed model in order to reduce the computational effort required to solve it. These constraints, which may be called cuts, restrict the feasible region of the binary variables, but do not eliminate the optimal solution.

\subsection{Additional Constraints}

For an EDS, without distributed generation and considering the radiality constraint, we obtain by definition that each node in the final solution is fed by one and only one branch. This condition is represented by constraint (23) for each node $j$, and it ensures that the sum of the binary variables that limit the active power flow in the forward direction $\left(y_{i j}^{+}\right)$and the binary variables that limit the active power flow in the backward direction $\left(y_{i j}^{-}\right)$is equal to one.

For example, assume that, in the case shown in Fig. 4, we have found that the branch $h j$ feeds the node $j$; therefore, $y_{h j}^{+}$ is equal to 1 . Consequently, other variables associated with flows that could feed node $j$, namely, $y_{j i}^{-}$and $y_{k j}^{+}$, should be equal to zero.

$$
\sum_{i j \in \Omega_{1}} y_{i j}^{+}+\sum_{j i \in \Omega_{1}} y_{j i}^{-}=1 \quad \forall j \in \Omega_{\mathrm{b}} .
$$

So, for this case, (23) will look as follows:

$y_{h j}^{+}+y_{k j}^{+}+y_{j i}^{-}=1$.

Another constraint is defined for nodes that can be connected only via two branches, which are represented by the set $\Omega_{c}$, as shown in Fig. 5 (node $i$ ). Assuming that there is a branch $k i$ with end node $i$, if the active power flow in the forward direction of branch $i j$ is greater than zero, then the active power flow in the forward direction of branch $k i$ is necessarily

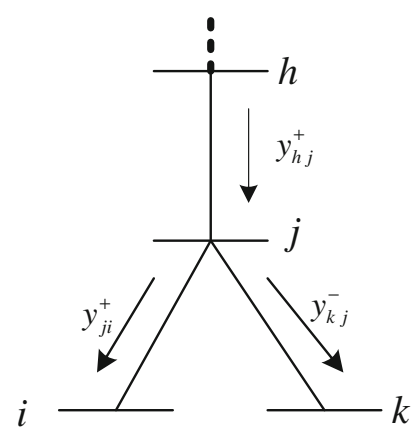

Fig. 4 Representation of partial EDS

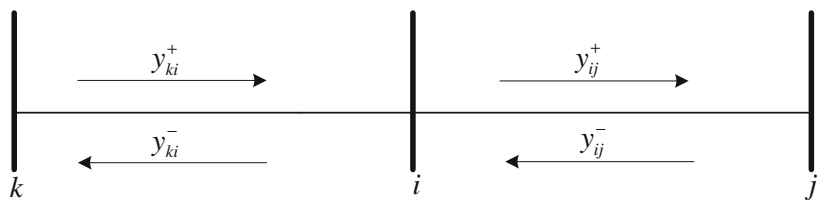

Fig. 5 Control of circuit state of EDS 
active. Similarly, for the active power flow in the backward direction of branch $k i$ to be greater than zero, it is required that the active power flow in the backward direction of branch $i j$ must be greater than zero; thus, $y_{i j}^{-}$has to be equal to one. So, for nodes in $\Omega_{\mathrm{c}}$ (24) can be written as

$$
\left\{\begin{array}{l}
y_{i j}^{+} \leq y_{k i}^{+} \\
y_{k i}^{-} \leq y_{i j}^{-}
\end{array} \quad \forall i \in \Omega_{\mathrm{c}} .\right.
$$

Additionally, an initial superior quota is added in the proposed methodology, which limits the objective function of the reconfiguration problem with a superior bound. This limit corresponds to the active power losses associated with the initial topology of the EDS. This initial value allows the Branch-and-Bound $(\mathrm{B} \& \mathrm{~B})$ algorithm to remove the nodes of the $\mathrm{B} \& \mathrm{~B}$ tree with an objective function greater than the initial active power losses. Consequently, it is possible to reduce the evaluated solutions during the search process of the $\mathrm{B} \& \mathrm{~B}$ algorithm.

\section{Results}

The proposed methodology was implemented in the mathematical programming language AMPL (Fourer et al. 2002) and was solved using the commercial software CPLEX (CPLEX 2008) with default options. All simulations were done using a computer with an Intel processor i7PC of 1.87 GHz. The test systems of 33 nodes (Chiou et al. 2005), 70 nodes (Chiang and Jean-Jumeau 1990), 136 nodes (Carreño et al. 2008), and 417 nodes (Ramírez and Bernal 1998) were used to show the performance and robustness of the proposed methodology. The operation point for the solution of the reconfiguration problem was compared with the one obtained using the load flow sweep method (AC-LF).

\subsection{3-Node Distribution System}

This system has 33 nodes and 37 branches, with a nominal voltage magnitude of $12.66 \mathrm{kV}$, supplying a total load of $3715 \mathrm{~kW}$ and $2315 \mathrm{kVAr}$. This EDS has 32 interconnection switches, normally closed, and 5 open interconnection switches. Table 1 presents the results found by the proposed methodology and the best results found in the literature. Note that the proposed methodology found the same solution as that reported in the specialized literature.
In the initial configuration of the system, the lower voltage magnitude is at node 17 with a value of 0.91309 pu. After the reconfiguration, the lower voltage magnitude is found at node 31 with a value of $0.93779 \mathrm{pu}$; that voltage drop does not violate the minimum voltage magnitude, which is $7 \%$. The computational time spent by the proposed methodology to find the optimal solution for the system was $4 \mathrm{~s}$; this time is required for the $\mathrm{B} \& \mathrm{~B}$ algorithm to explore the entire search tree.

\subsection{0-Node Distribution System}

This system has a 69 load nodes, one substation, and 74 branches. The nominal voltage magnitude is $12.66 \mathrm{kV}$, supplying a total load of $1117.86 \mathrm{~kW}$ and $902.25 \mathrm{kVAr}$. This system has 5 interconnection switches that are initially open. Table 2 presents the results found by the proposed methodology and the best results found in the literature. Note that the proposed methodology found the same solution as that reported in the specialized literature.

In the initial configuration of the system, the lower voltage magnitude is found at node 66 with a value of 0.971963 pu. After the reconfiguration, the lower voltage magnitude is found at node 62 with a value of $0.982399 \mathrm{pu}$. The computational time spent by the proposed methodology to find the optimal solution for the system was $8 \mathrm{~s}$; this time is required for the B\&B algorithm to explore the entire search tree.

\subsection{6-Node Distribution System}

This system has 136 nodes and 156 branches. The nominal voltage magnitude is $13.8 \mathrm{kV}$, supplying a total load of $18313.809 \mathrm{~kW}$ and $9384.827 \mathrm{kVAr}$. This system has 21 interconnection switches initially open. The results found by the proposed methodology and the best results found in the literature are shown in Table 3.

Note that the result found by the proposed method, even utilizing distinct values for the constant $S$, are the same as that reported in the specialized literature, as can be seen in Carreño et al. (2008). The computational time spent by the proposed methodology to find the optimal solution for the system utilizing $S=0$ was $13 \mathrm{~s}$ and utilizing $S=1$ was $1,236 \mathrm{~s}$; this time is required for the $\mathrm{B} \& \mathrm{~B}$ algorithm to explore the entire search tree, but the solution was found in $363 \mathrm{~s}$. After the reconfiguration, the lowest voltage magnitude in the system is 0.9589 pu at node 106 , whose voltage drop does not violate the minimum voltage magnitude of $5 \%$. In the initial
Table 1 Results obtained for 33-node system

\begin{tabular}{llll}
\hline Methodology & $\begin{array}{l}\text { Open interconnection } \\
\text { switches }\end{array}$ & $\begin{array}{l}\text { Active power } \\
\text { losses (kW) }\end{array}$ & $\begin{array}{l}\text { Active power losses } \\
\text { using the AC-LF (kW) }\end{array}$ \\
\hline Proposed & $7,9,14,32,37$ & 139.74 & 139.55 \\
Mantovani et al. (2000) & $7,9,14,32,37$ & 139.55 & 139.55
\end{tabular}


Table 2 Results obtained for 70-node system

\begin{tabular}{llll}
\hline Methodology & $\begin{array}{l}\text { Open interconnection } \\
\text { switches }\end{array}$ & $\begin{array}{l}\text { Active power losses } \\
(\mathrm{kW})\end{array}$ & $\begin{array}{l}\text { Active power losses } \\
\text { using the AC-LF (kW) }\end{array}$ \\
\hline Proposed & $15,59,62,70,71$ & 9.95 & 9.34 \\
Mantovani et al. (2000) & $15,59,62,70,71$ & 9.34 & 9.34 \\
\hline
\end{tabular}

Table 3 Results obtained for 136-node system

\begin{tabular}{|c|c|c|c|c|c|}
\hline Methodology & $\begin{array}{l}\text { Open interconnection } \\
\text { switches }\end{array}$ & S & Time (s) & $\begin{array}{l}\text { Active power losses } \\
(\mathrm{kW})\end{array}$ & $\begin{array}{l}\text { Active power losses } \\
\text { using the AC-LF }(\mathrm{kW})\end{array}$ \\
\hline Proposed & $\begin{array}{l}7,35,51,90,96,106,118,126,135,137 \\
138,141,142,144,145,146,147,148,150 \\
151,155\end{array}$ & 0 & 13 & 284.35 & 280.14 \\
\hline Proposed & $\begin{array}{l}7,35,51,90,96,106,118,126,135,137 \\
138,141,142,144,145,146,147,148,150, \\
151,155\end{array}$ & 1 & 1236 & 280.51 & 280.14 \\
\hline Carreño et al. (2008) & $\begin{array}{l}7,35,51,90,96,106,118,126,135,137 \\
138,141,142,144,145,146,147,148,150 \\
151,155\end{array}$ & - & 0.4 & 280.14 & 280.14 \\
\hline
\end{tabular}

Table 4 Results obtained for the 417-node system

\begin{tabular}{|c|c|c|c|c|c|}
\hline Methodology & $\begin{array}{l}\text { Open interconnection } \\
\text { switches }\end{array}$ & $\mathrm{S}$ & Time (s) & $\begin{array}{l}\text { Active power losses } \\
(\mathrm{kW})\end{array}$ & $\begin{array}{l}\text { Active power losses } \\
\text { using the AC-LF }(\mathrm{kW})\end{array}$ \\
\hline Proposed & $\begin{array}{l}5,13,15,16,21,26,31,53,54,59,60,73,82, \\
87,94,96,97,110,111,115,136,142,150 \\
155,156,163,165,168,169,178,179,191 \\
195,199,214,221,254,256,266,282,317 \\
322,325,358,362,369,392,395,403,404 \\
416,423,426,431,436,437,446,449,466\end{array}$ & 0 & 7,445 & 591.79 & 581.80 \\
\hline Proposed & $\begin{array}{l}5,13,15,16,21,26,31,54,57,59,60,73,86, \\
87,94,96,97,110,111,115,136,142,149, \\
150,155,156,163,168,169,178,179,191, \\
195,199,214,221,254,256,266,282,317 \\
322,325,358,359,362,369,392,395,403 \\
404,416,423,426,431,436,437,446,449\end{array}$ & 1 & 171,425 & 585.65 & 581.57 \\
\hline Lavorato et al. (2012) & $\begin{array}{l}5,13,15,16,21,26,31,54,57,59,60,73,86, \\
87,94,96,97,110,111,115,136,142,149, \\
150,155,156,163,168,169,178,179,191, \\
195,199,214,221,254,256,266,282,317 \\
322,325,358,359,362,369,392,395,403 \\
404,416,423,426,431,436,437,446,449\end{array}$ & - & - & 685.88 & 685.88 \\
\hline
\end{tabular}

configuration of the system, the lower voltage magnitude is $0.9326 \mathrm{pu}$ at node 106 . Thus, the reconfiguration of the system improved the voltage magnitude profile of the system.

\subsection{7-Node Distribution System}

This system has 415 nodes and 473 branches and is a largescale EDS. The nominal voltage magnitude is $10.0 \mathrm{kV}$, supplying a total load of $27958.01 \mathrm{~kW}$ and $13736.81 \mathrm{kVAr}$. This system has 59 interconnection switches initially open. The results found by the proposed methodology and the best results found in the literature are shown in Table 4.

The result found by the proposed methodology, utilizing different values for the constant $S$ for this system, is better than the results reported in the specialized literature, as can be seen in Lavorato et al. (2012). The computational time spent by the proposed methodology to find the optimal solution for this system, which is the time needed by the B\&B algorithm to explore the entire search tree, was $7,445 \mathrm{~s}$ for $S=0$, but the optimal solution was found in $1,958 \mathrm{~s}$. For $S=1$, the proposed methodology found the optimal solution in $171,425 \mathrm{~s}$. After the reconfiguration, the lowest voltage magnitude in the system is $0.954233 \mathrm{pu}$ at node 48 , whose voltage drop does not violate the minimum voltage magnitude of $5 \%$. When the parameter $S$ is increased, the proposed methodology finds an optimal solution of better quality using more computational time, because the constant $S$ increases the number of binary variables of the model. This difference between the solutions 
found by the proposed method is irrelevant when compared with the computational effort employed to find the optimal solution using $S=1$.

\section{Conclusions}

In this paper, the reconfiguration problem of electrical distribution systems has been modeled as an MINLP model, with the objective of reducing the active power losses of the EDS. Two linearization techniques were used to obtain an equivalent MILP model. Additional constraints are included in the proposed model to reduce the computational effort demanded by its solution. The use of an MILP model guarantees convergence to optimality using conventional MILP solvers. The solutions obtained using the proposed methodology for systems of $33,70,136$, and 417 nodes are equal to or better than the solutions found in the specialized literature. The results show that the power losses are calculated with great precision in comparison with the load flow sweep method.

\section{Appendix}

\subsection{Illustrative Example of the Power Balance Formulation}

In order to clarify the formulation for the balance of active and reactive power used in this study, a test system of 3 nodes will be used (Fig. 6).

The active power losses of the circuit 2-3 represented by $R_{23} I_{23}^{2}$ are used in the balance of active power of the node 2 as follows:

$$
P_{12}-\left(P_{23}+R_{23} I_{23}^{2}\right)+P_{2}^{\mathrm{S}}-P_{2}^{\mathrm{D}}=0
$$

Thus, we can proceed to represent the active power balance in node 2 , considering the node power injections: supplied $\left(P_{2}^{\mathrm{S}}\right)$ and demanded $\left(P_{2}^{\mathrm{D}}\right)$ powers, incoming power flows from previous branches $\left(P_{12}\right)$, and power flows from posterior branches $\left(P_{23}\right)$ plus their power losses $\left(R_{23} I_{23}^{2}\right)$. The same representation is made for the reactive power balance in which the reactive power losses are represented by $X_{23} I_{23}^{2}$. The reactive power balance is described below:

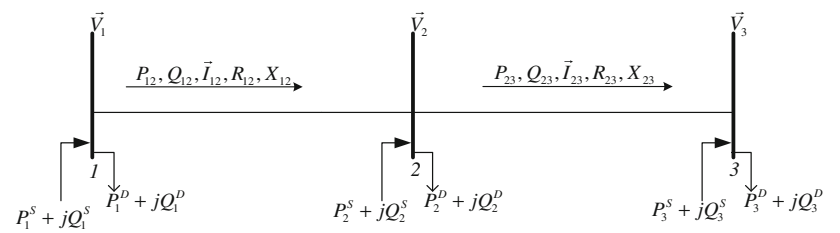

Fig. 6 Illustrative test system

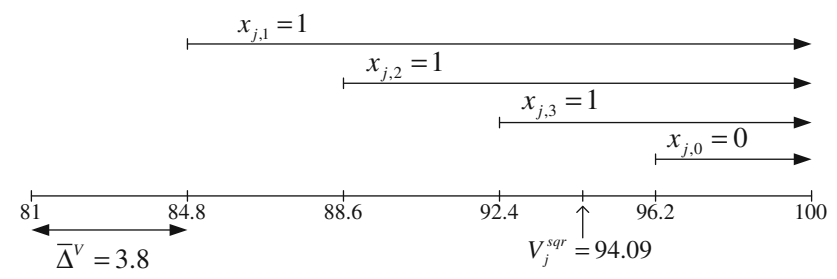

Fig. 7 Illustrative example of the voltage discretization

$Q_{12}-\left(Q_{23}+X_{23} I_{23}^{2}\right)+Q_{2}^{\mathrm{S}}-Q_{2}^{\mathrm{S}}=0$

6.2 Illustrative Example of the Discretization of the Square of the Voltage Magnitude

Consider the following data: $V_{j}^{\mathrm{sqr}}=94.09 k V^{2} ; I_{i j}^{\mathrm{sqr}}=$ $62500 A^{2} ; \underline{V}=9 k V ; \bar{V}=10 k V$; and $S=4$, the constant $\bar{\Delta}^{V}=3.8 k V^{2}$ can be obtained. The Fig. 7 shows an illustrative example of the discretization of the square of the voltage magnitude. Using Eq. (16), the values of the binary variables $x_{j, S}$ can be established. So, $x_{j, 1}=x_{j, 2}=x_{j, 3}=1$ and $x_{j, 4}=0$.

From Eq. (17), one can find the values of $P_{i j, s}^{c}$.

$P_{i j, 1}^{\mathrm{c}}=P_{i j, 2}^{\mathrm{c}}=P_{i j, 3}^{\mathrm{c}}=\bar{\Delta}^{V} I_{i j}^{\mathrm{sqr}}=237500$ and $P_{i j, 4}^{\mathrm{c}}=0$

Therefore, from (18), it is concluded that

$\left(V^{2}+\frac{\bar{\Delta}^{V}}{2}\right) I_{i j}^{\mathrm{sqr}}+\sum_{s=1}^{4} P_{i j, s}^{\mathrm{c}}=5893750 V A^{2}$.

While the exact value is

$V_{j}^{\mathrm{sqr}} I_{i j}^{\mathrm{sqr}}=(94.09) 62500=5880625 V A^{2}$.

Note that the result of the linear approximation for this example has an error of $0.22 \%$ compared with the exact value. This error can be reduced increasing the value of $S$.

\subsection{Illustrative Example of the Piecewise Linear Modeling of $P_{i j}^{2}$}

The Fig. 8 shows an illustrative example of the modeling of $P_{i j}^{2}$ as a piecewise linear function. Consider the following data: $P_{i j}=60 k W, V^{\text {nom }} \bar{I}_{i j}=90 k V A$, and $W=5$. Thus, one can obtain $\bar{\Delta}_{i j}^{\mathrm{S}}=18 \mathrm{kVA}$ from (22), and the values of $m_{i j, w}^{\mathrm{S}}$ can be calculated from (21)

$$
\begin{gathered}
m_{i j, 1}^{\mathrm{S}}=18, \quad m_{i j, 2}^{\mathrm{S}}=54, \quad m_{i j, 3}^{\mathrm{S}}=90, \\
m_{i j, 4}^{\mathrm{S}}=126 \text { e } m_{i j, 5}^{\mathrm{S}}=162 .
\end{gathered}
$$

From Eq. (20b), one can find the values of the variables $\Delta_{i j, w}^{\mathrm{P}}$ : 


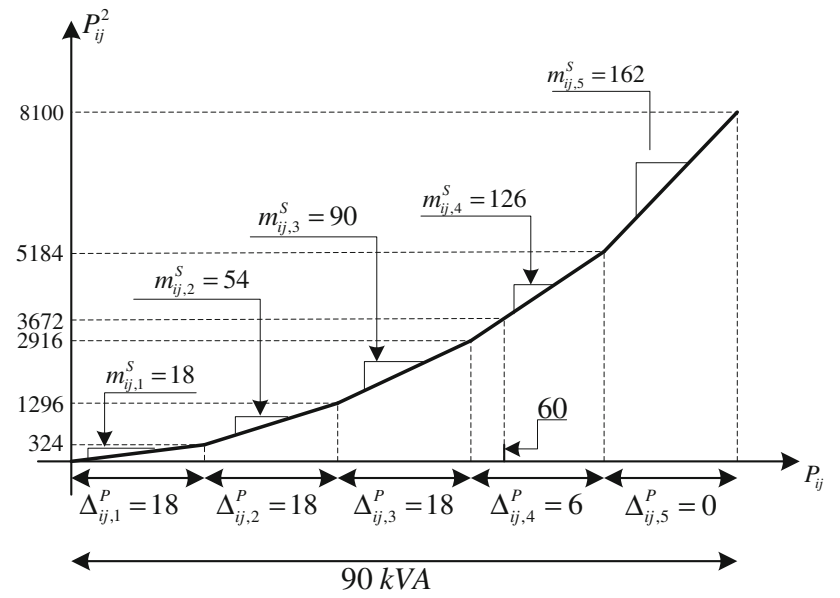

Fig. 8 Illustrative example of the linearization

$\Delta_{i j, 1}^{\mathrm{P}}=\Delta_{i j, 2}^{\mathrm{P}}=\Delta_{i j, 3}^{\mathrm{P}}=18, \quad \Delta_{i j, 4}^{\mathrm{P}}=6 \mathrm{e} \Delta_{i j, 5}^{\mathrm{P}}=0$.

It is concluded that $\sum_{w=1}^{W} m_{i j, w}^{\mathrm{S}} \Delta_{i j, w}^{\mathrm{P}}=3672 k W^{2}$. The exact value for $P_{i j}^{2}$ is (60) $60=3600 k W^{2}$.

Note that the result of modeling $P_{i j}^{2}$ as a piecewise linear function has an error of $2 \%$ when compared to the exact value. This error can be reduced by increasing the value of $W$.

\section{References}

Abul'Wafa, A. R. (2011). A new heuristic approach for optimal reconfiguration in distribution systems. Electric Power Systems Research, 81(2), 282-289.

Abur, A. (1996). A modified linear programming method for distribution system reconfiguration. Electric Power Energy Systems Research, 18(7), 469-474.

Baran, M. E., \& Wu, F. F. (1989). Network reconfiguration in distribution systems for loss reduction and load balancing. IEEE Transactions on Power Delivery, 4(2), 1401-1407.

Carreño, E., Romero, R., \& Feltrin, A. P. (2008). An efficient codification to solve distribution network reconfiguration for loss reduction problem. IEEE Transactions on Power Systems, 23(4), 1542-1551.

Cespedes, R. (1990). New method for the analysis of distribution networks. IEEE Transactions on Power Delivery, 5(1), 391-396.

Chang, C. F. (2008). Reconfiguration and capacitor placement for loss reduction of distribution systems by ant colony search algorithm. IEEE Transactions on Power Systems, 23(4), 1747-1755.

Chiang, H. D., \& Jean-Jumeau, R. (1990). Optimal network reconfigurations in distribution systems: Part 2: Solution algorithms and numerical results. IEEE Transactions on Power Delivery, 5(3), 1568-1574.

Chiou, J. P., Chang, C. F., \& Su, C. T. (2005). Variable scaling hybrid differential evolution for solving network reconfiguration of distribution systems. IEEE Transactions on Power Systems, 20(2), 668-674.

CPLEX (2008). Optimization subroutine library guide and reference, version 11.0, CPLEX Division, ILOG Inc., Incline Village, NV, USA.

Fourer, R., Gay, D. M., \& Kernighan, B. W. (2002). AMPL: A modeling language for mathematical programming. Pacific Grove: Duxbury.

Franco, J. F., Rider, M. J., Lavorato, M., \& Romero, R. (2013a). Optimal conductor size selection and reconductoring in radial distribution systems using a mixed-integer LP approach. IEEE Transactions on Power Systems, 28(1), 10-20.
Franco, J. F., Rider, M. J., Lavorato, M., \& Romero, R. (2013b). A mixed-integer LP model for the optimal allocation of voltage regulators and capacitors in radial distribution systems. International Journal of Electrical Power \& Energy Systems, 48, 123-130.

Goswami, S. K., \& Basu, S. K. (1992). A new algorithm for the reconfiguration of distribution feeders for loss minimization. IEEE Transactions on Power Delivery, 7(3), 1484-1491.

Guimarães, M. A. N., Lorenzeti, J. F. C., \& Castro C. A. (2004). Reconfiguration of distribution system for voltage stability margin enhancement using tabu search.In POWERCON 2004: International Conference on Power System Technology (pp. 1556-1561). Singapore.

Hayashi, Y., Iwamoto, S., Furuya, S., \& Liu, C. C. (1996). Efficient determination of optimal radial power system structure using hopfield neural network with constrained noise. IEEE Transactions on Power Delivery, 11(3), 1529-1535.

Jabr, R. A., Singh, R., \& Pal, B. C. (2012). Minimum loss network reconfiguration using mixed-integer convex programming. IEEE Transactions on Power Systems, 27(2), 1106-1115.

Kangan, N., \& Barioni, C. C. O. (1999). Fuzzy decision model for the reconfiguration of distribution networks using genetic algorithms. In Proceedings of the 13th Power System Computation Conference (pp. 929-935). Trondheim: Norway..

Lavorato, M., Franco, J. F., Rider, M. J., \& Romero, R. (2012). Imposing radiality constraints in distribution system optimization problems. IEEE Transactions on Power Systems, 11(3), 172-180.

Mantovani, J. R. S., Casari, F., \& Romero, R. A. (2000). Reconfiguração de sistemas de distribuição radiais utilizando o critério de queda de tensão (Reconfiguration of radial distribution systems using the criterion of voltage drop). SBA Controle \& Automação, 11, 150-159.

Martin, J. A., \& Gil, A. J. (2008). A new heuristic approach for distribution systems loss reduction. Electric Power Systems Research, 78(11), 1953-1958.

Merlin, A., \& Back, H. (1975). Search for a minimal-loss operating spinning tree configuration in an urban power distribution system. In Power System Computation Conference (pp. 1-18).

Raju, G., \& Bijwe, P. R. (2008). An efficient algorithm for minimum loss reconfiguration of distribution system based on sensitivity and heuristics. IEEE Transactions on Power Systems, 23(3), 1280-1287.

Ramírez, I. J. R., \& Bernal, J. L. A. (1998). Genetic algorithms applied to the design of large power distribution systems. IEEE Transactions on Power Systems, 13(2), 696-703.

Ramos, E. R., Exposito, A. G., Santos, J. R., \& Iborra, F. L. (2005). Path-based distribution network modelling: Application to reconfiguration for loss reduction. IEEE Transactions Power Systems, 20(2), 556-564.

Romero-Ramos, E., Riquelme-Santos, J., \& Reyes, J. (2010). A simpler and exact mathematical model for the computation of the minimal power losses tree. Electric Power Systems Research, 80(5), 562-571.

Salazar, H., Gallero, R., \& Romero, R. (2006). Artificial neural networks and clustering techniques applied in the reconfiguration of distribution systems. IEEE Transactions on Power Delivery, 21(3), $1735-1742$.

Schmidt, H. P., Ida, N., Kagan, N., \& Guaraldo, J. C. (2005). Fast reconfiguration of distribution systems considering loss minimization. IEEE Transactions on Power Systems, 20(3), 1311-1319.

Shirmohammadi, D., Hong, H. W., Semlyen, A., \& Luo, G. X. (1988). A compensation based power flow method for weakly meshed distribution and transmission networks. IEEE Transactions on Power Systems, 3(2), 753-762.

Zhu, J. Z. (2002). Optimal reconfiguration of electrical distribution network using the refined genetic algorithm. Electric Power Systems Research, 62(1), 37-42. 